Open Access

\title{
Adding anthropometric measures of regional adiposity to BMI improves prediction of cardiometabolic, inflammatory and adipokines profiles in youths: a cross-sectional study
}

Hanen Samouda ${ }^{1 *}$, Carine de Beaufort ${ }^{2}$, Saverio Stranges ${ }^{1}$, Benjamin C. Guinhouya ${ }^{3}$, Georges Gilson ${ }^{4}$, Marco Hirsch ${ }^{5}$, Julien Jacobs ${ }^{1}$, Sonia Leite ${ }^{6}$, Michel Vaillant ${ }^{6}$ and Frédéric Dadoun ${ }^{1,7}$

\begin{abstract}
Background: Paediatric research analysing the relationship between the easy-to-use anthropometric measures for adiposity and cardiometabolic risk factors remains highly controversial in youth. Several studies suggest that only body mass index (BMI), a measure of relative weight, constitutes an accurate predictor, whereas others highlight the potential role of waist-to-hip ratio (WHR), waist circumference (Waist C), and waist-to-height ratio (WHtR). In this study, we examined the effectiveness of adding anthropometric measures of body fat distribution (Waist C Z Score, WHR Z Score and/or WHtR) to BMI Z Score to predict cardiometabolic risk factors in overweight and obese youth. We also examined the consistency of these associations with the "total fat mass + trunk/legs fat mass" and/or the "total fat mass + trunk fat mass" combinations, as assessed by dual energy X-ray absorptiometry (DXA), the gold standard measurement of body composition.
\end{abstract}

Methods: Anthropometric and DXA measurements of total and regional adiposity, as well as a comprehensive assessment of cardiometabolic, inflammatory and adipokines profiles were performed in 203 overweight and obese 7-17 year-old youths from the Paediatrics Clinic, Centre Hospitalier de Luxembourg.

Results: Adding only one anthropometric surrogate of regional fat to BMI Z Score improved the prediction of insulin resistance (WHR Z Score, $R^{2}: 45.9 \%$. Waist C Z Score, R²: $45.5 \%$ ), HDL-cholesterol (WHR Z Score, R²: $9.6 \%$. Waist C Z Score, R': $10.8 \%$. WHtR, R2: $6.5 \%$ ), triglycerides (WHR Z Score, R': $11.7 \%$. Waist C Z Score, R2: $12.2 \%$ ), adiponectin (WHR Z Score, R²: $14.3 \%$. Waist C Z Score, R²: $17.7 \%$ ), CRP (WHR Z Score, $R^{2}: 18.2 \%$. WHtR, R²: $23.3 \%$ ), systolic (WHtR, $\left.R^{2}: 22.4 \%\right)$, diastolic blood pressure (WHtR, $\left.R^{2}: 20 \%\right)$ and fibrinogen (WHtR, $\left.R^{2}: 21.8 \%\right)$. Moreover, WHR Z Score, Waist C Z Score and/or WHtR showed an independent significant contribution according to these models. These results were in line with the DXA findings.

Conclusions: Adding anthropometric measures of regional adiposity to BMI Z Score improves the prediction of cardiometabolic, inflammatory and adipokines profiles in youth.

Keywords: Obesity, Overweight, Body mass index, Body fat distribution, DXA, Anthropometry

\footnotetext{
* Correspondence: hanene.samouda@lih.lu

${ }^{1}$ Population Health Department, Epidemiology and Public Health Research Unit, Luxembourg Institute of Health, 1A-B, rue Thomas Edison, L-1445

Strassen, Luxembourg

Full list of author information is available at the end of the article
} 


\section{Background}

Several studies have focused on the presence of early biological abnormalities in excess-weight youths, including elevated fasting glycaemia, insulin resistance, hypertriglyceridemia, high-density lipoprotein cholesterol (HDLcholesterol), elevated blood pressure and causing several comorbidities in adults [1-5]. Furthermore, some adipokines, namely leptin, adiponectin and resistin, have been identified as potential risk markers for a systemic low-grade inflammation state, which might lead to insulin resistance, type-2 diabetes and cardiovascular (CV) diseases [6-8].

Moreover, beyond global excess weight, the role of the abdominovisceral adiposity as independent cardiometabolic risk factor has been underlined from children onwards [9], while more peripheral fat has been considered as protective [10].

Magnetic Resonance Imaging (MRI), Computed Tomography-Scan (CT-Scan) and Dual-energy X ray Absorptiometry (DXA) have been described as the gold standard of adiposity measurement and used to accurately assess body fat distribution and related comorbidities [9, 11, 12]. However, these techniques are still no accessible because of their high cost and irradiation in the case of CT-Scan measurements as well [11, 12].

Therefore, in order to assess the comorbidities associated with overweight and obesity and abdomino-visceral adiposity in youths, the identification of simple and accurate anthropometric methods that can be used with efficiency as clinical and research tools is essential.

Studies analysing the relationship between the easy-touse anthropometric measures for total fat mass, body fat distribution and cardiometabolic risk factors are highly controversial when it comes to youths. Several authors suggested that only body mass index (BMI) constitutes an accurate predictor of biological abnormalities and cardiometabolic impairments [13-17], whereas others highlighted the role of the waist-to-hip ratio (WHR) $[18,19]$, waist circumference (Waist C) $[20,21]$ and/or waist-to-height ratio (WHtR) [22, 23]. Furthermore, certain studies showed no significant differences in the ability of BMI and WHR [24], BMI and Waist C [25], BMI and WHtR [26], as well as Waist C and WHtR [27] to predict cardiometabolic risk factors. Finally, in some other studies, differential associations were observed between CV risk factors and anthropometric measures $[28,29]$.

In adults, extensive studies showed that adding anthropometric measures of body fat distribution such as WHR or Waist C, to BMI, allows predicting CV risk factors, diseases and death more accurately [2, 30-34]. This type of associations has not really been developed in paediatric populations. Indeed, in an attempt to predict cardiometabolic risk factors in youths, some previous paediatric studies either tested the efficiency of a single anthropometric measurement $[14,21,23,25]$ or assessed the contribution of BMI only as a potential confounder of other variables involved [18, 20, 27, 29].

The present study investigated the ability of the "BMI and Waist C", "BMI and WHR" and/or "BMI and WHtR" associations to predict cardiometabolic risk factors in overweight and obese youths. The consistency of our findings was evaluated by assessing the ability to predict the same risk factors presented by the associations between total fat mass and trunk fat mass, respectively total fat mass and trunk/legs fat mass as obtained by dual energy X-ray absorptiometry (DXA), which is the body-composition gold-standard analysis.

\section{Methods}

\section{Participants}

Two hundred three overweight and/or obese children (52.2 \% female) according to the IOTF definition [35], aged 7 to17 years old, and visiting the Diabetes $\mathcal{E}$ Endocrinology Care Paediatrics Clinic, Centre Hospitalier de Luxembourg, were invited to participate in a crosssectional study performed between September 2006 and June 2008. The parents gave their written informed consent. The study was approved by the National Ethics Committee and authorized by the National Commission for Data Protection in Luxembourg.

\section{Anthropometry and body composition}

Height, weight, waist and hip circumferences have been performed according to the recommendations of Lohmann [36]. BMI, WHR and WHtR ratios were calculated. Total, trunk and legs fat masses were measured by DXA using the Hologic QDR4500W densitometer. Trunk/legs fat mass index was calculated.

\section{Clinical and biological measurements}

Blood pressures was measured with an aneroid sphygmomanometer (Welch AL) in the sitting position: 3 readings were performed and the average was retained. Systolic blood pressure (SBP) and diastolic blood pressure (DBP) Z Scores were calculated according to the formula proposed by The Fourth Report on the Diagnosis, Evaluation, and Treatment of High Blood Pressure in Children and Adolescents [37]. Roche reagents on a P module of Roche Modular (Basel, Switzerland) were used to assess fasting glucose, triglycerides, HDL-cholesterol and lowdensity lipoprotein cholesterol (LDL-cholesterol). An Olympus latex reagent was used on the same $\mathrm{P}$ module of a Roche Modular to measure C-reactive protein (CRP). A chimiluminescent assay on Siemens Immulite 2000 (Deerfield, USA) was used to measure fasting insulin. Fibrinogen was assessed on Stago Compact (Asnières sur Seine, France). ELISA kits provided by Mediagnost (Reutlingen, Germany) were used to assess leptin, adiponectin and resistin. We also calculated the homeostasis 
model assessment of insulin resistance [HOMA IR = fasting insulin $(\mu \mathrm{U} / \mathrm{ml}) \times$ fasting glucose $(\mathrm{mmol} / \mathrm{l}) / 22.5]$ [38] and the quantitative insulin sensitivity check index [QUICKI index $=1 /(\log$ fasting insulin in $\mu \mathrm{U} / \mathrm{ml}+\log$ glucose in $\mathrm{mg} / \mathrm{dl})$ ] [39]. Tanner stages were assessed [40, 41].

\section{Statistical analyses}

The combination of the Kolmogorov-Smirnov test and of the Lilliefors' test was used to check the normal data distribution. Triglycerides, HDL cholesterol, fasting insulin, HOMA IR, CRP, fibrinogen, adiponectin, leptin and resistin were log transformed (skewed variables).

Mean \pm sd and/or percentages were calculated and compared by the Student's $t$ test (descriptive data). In the absence of national L,M,S data, BMI, Waist $\mathrm{C}$ and WHR Z Scores, as well as the overweight (boys: $\geq 91$ th percentile; girls: $\geq 89$ th percentile) and obesity ( $\geq 99$ th percentile) thresholds, were defined according to the L, M, S Dutch values [42, 43] and the IOTF definition [35].

\section{Anthropometric and DXA prediction of cardiometabolic risk factors}

To test the ability of each single anthropometric variable to predict the risk factors, the univariate linear analysis [Pearson's R] was used. To assess the combined effect of the "BMI and Waist C", "BMI and WHR", "BMI and WHtR" as well as "total and trunk fat masses" and "total and trunk/legs fat masses" associations on the prediction of the risk factors, multivariable linear analyses were performed. An additional effect of Waist C Z Score, WHR Z Score and/or WHtR was highlighted when 1.the global variance of the model $\left(R^{2}\right)$ was improved and 2.the variable showed an independent significant contribution to the model (significant $r_{\text {partial }}^{2}$ ), independently of the BMI $\mathrm{Z}$ Score. All models were age-, sex- and pubertal status adjusted. To test the consistency of the anthropometry and DXA findings, similar analyses were performed to assess the potential additional impact of the trunk fat mass and/or the trunk/legs fat index, beyond the total fat mass. Results with a $p$-value $<0.05$ were considered statistically significant. Statistical analyses were performed using SPSS for Windows Version 17.0.

\section{Results}

The anthropometric, body composition and biological characteristics of the participants are summarized in Table 1.

\section{Relationships between single anthropometric variables and CV risk factors}

BMI Z Score was the most accurate single predictor of fasting glucose, fasting insulin, HOMA IR, QUICKI,
Table 1 Subject characteristics

\begin{tabular}{|c|c|c|c|}
\hline & Girls & Boys & All children \\
\hline $\bar{N}$ & 106 & 97 & 203 \\
\hline Age (years) & $12.2 \pm 2.5$ & $11.8 \pm 2.4$ & $12.0 \pm 2.4$ \\
\hline \multicolumn{4}{|l|}{ Pubertal status (Percentages) } \\
\hline Yes & $84(79.2 \%)$ & 47 (48.5 \%) & $131(64.5 \%)$ \\
\hline No & $22(20.8 \%)$ & $50(51.5 \%)$ & $72(35.5 \%)$ \\
\hline \multicolumn{4}{|l|}{ Anthropometry } \\
\hline $\mathrm{BMI}\left(\mathrm{kg} / \mathrm{m}^{2}\right)$ & $28.5 \pm 5.6$ & $28.2 \pm 4.9$ & $28.3 \pm 5.3$ \\
\hline BMI Z score & $2.42 \pm 0.58$ & $2.68 \pm 0.53^{*}$ & $2.54 \pm 0.57$ \\
\hline Waist C (cm) & $83.8 \pm 12.4$ & $86.5 \pm 11.5$ & $85.1 \pm 12.0$ \\
\hline Waist C Z score & $2.22 \pm 0.63$ & $2.46 \pm 0.58^{*}$ & $2.33 \pm 0.62$ \\
\hline$W H t R^{a}$ & $0.54 \pm 0.06$ & $0.56 \pm 0.05^{*}$ & $0.55 \pm 0.06$ \\
\hline$W H R^{b}$ & $0.84 \pm 0.06$ & $0.89 \pm 0.05^{* *}$ & $0.86 \pm 0.06$ \\
\hline WHR Z score & $0.71 \pm 0.89$ & $0.85 \pm 0.95$ & $0.78 \pm 0.92$ \\
\hline \multicolumn{4}{|l|}{ Biology } \\
\hline Fasting glucose (mg/dl) & $86.2 \pm 6.8$ & $86.9 \pm 6.2$ & $86.5 \pm 6.5$ \\
\hline Fasting insulin (mUI/l) & $17.5 \pm 8.5$ & $14.8 \pm 8.3^{*}$ & $16.2 \pm 8.5$ \\
\hline HOMA IR & $3.76 \pm 1.98$ & $3.21 \pm 1.87^{*}$ & $3.50 \pm 1.94$ \\
\hline QUICKI & $0.321 \pm 0.024$ & $0.330 \pm 0.027^{*}$ & $0.326 \pm 0.026$ \\
\hline Triglycerides (mg/dl) & $98.4 \pm 58.4$ & $90.0 \pm 51.1$ & $94.3 \pm 55.1$ \\
\hline HDL cholesterol (mg/dl) & $54.4 \pm 12.7$ & $52.9 \pm 12.1$ & $53.7 \pm 12.4$ \\
\hline LDL cholesterol (mg/dl) & $92.3 \pm 29.0$ & $93.0 \pm 28.2$ & $92.6 \pm 28.6$ \\
\hline CRP (mg/l) & $2.9 \pm 4.1$ & $3.2 \pm 3.8$ & $3.1 \pm 4.0$ \\
\hline Fibrinogen (g/l) & $3.7 \pm 0.7$ & $3.6 \pm 0.6$ & $3.6 \pm 0.7$ \\
\hline Adiponectin $(\mu \mathrm{g} / \mathrm{ml})$ & $8.0 \pm 4.7$ & $7.8 \pm 4.5$ & $7.9 \pm 4.6$ \\
\hline Leptin (ng/ml) & $38.7 \pm 23.1$ & $27.4 \pm 16.1^{* *}$ & $33.3 \pm 20.8$ \\
\hline Resistin (ng/ml) & $5.3 \pm 2.2$ & $5.1 \pm 2.0$ & $5.2 \pm 2.1$ \\
\hline \multicolumn{4}{|l|}{ DXA } \\
\hline Total fat mass $(\mathrm{kg})$ & $32.51 \pm 14.29$ & $30.11 \pm 10.85$ & $31.37 \pm 12.80$ \\
\hline Trunk fat mass (kg) & $15.07 \pm 7.14$ & $14.17 \pm 5.87$ & $14.64 \pm 6.57$ \\
\hline Trunk/legs fat mass index & $1.22 \pm 0.24$ & $1.27 \pm 0.28$ & $1.24 \pm 0.26$ \\
\hline \multicolumn{4}{|l|}{ Blood pressure } \\
\hline $\mathrm{SBP}(\mathrm{mmHg})$ & $117 \pm 12$ & $118 \pm 14$ & $117 \pm 13$ \\
\hline SBP Z score & $0.99 \pm 1.04$ & $0.91 \pm 1.10$ & $0.95 \pm 1.07$ \\
\hline $\mathrm{DBP}(\mathrm{mmHg})$ & $71 \pm 9$ & $72 \pm 8$ & $72 \pm 9$ \\
\hline DBP Z score & $0.75 \pm 0.78$ & $0.81 \pm 0.64$ & $0.78 \pm 0.71$ \\
\hline
\end{tabular}

Data are $\mathrm{N}$ and/or means $\pm \mathrm{SD}$

${ }^{*} P$-value $<0.05 ;{ }^{* *} P$-value $<0.001$ for sex difference

aWHtR (waist to height ratio)

${ }^{b}$ WHR (waist-to-hip ratio)

leptin and resistin. Triglycerides and HDL cholesterol were most accurately predicted by Waist C Z Score. Blood pressure, CRP and fibrinogen were most accurately predicted by WHtR. WHR Z Score was the most accurate single predictor of adiponectin (Table 2). 
Table 2 Relationships between a single anthropometric measurement and biological variables

\begin{tabular}{|c|c|c|c|c|}
\hline Variable & BMI Z score & Waist C Z score & $\mathrm{WHtR}$ & WHR Z score \\
\hline \multicolumn{5}{|l|}{ Pearson's R } \\
\hline Fasting glucose & $0.235^{*}$ & $0.176^{*}$ & $0.193^{*}$ & 0.057 \\
\hline Fasting insulin ${ }^{a}$ & $0.490^{* *}$ & $0.483^{* *}$ & $0.463^{* *}$ & $0.295^{* *}$ \\
\hline HOMA IR ${ }^{\mathrm{a}}$ & $0.493^{* *}$ & $0.480^{* *}$ & $0.463^{* *}$ & $0.290^{* *}$ \\
\hline QUICKI & $-0.475^{* *}$ & $-0.463^{* *}$ & $-0.444^{* *}$ & $-0.283^{* *}$ \\
\hline Triglycerides $^{a}$ & $0.205^{*}$ & $0.270^{* *}$ & $0.250^{* *}$ & $0.249^{* *}$ \\
\hline HDL cholesterol ${ }^{a}$ & $-0.205^{*}$ & $-0.293^{* *}$ & $-0.252^{* *}$ & $-0.273^{* *}$ \\
\hline LDL cholesterol & -0.047 & -0.013 & 0.003 & 0.018 \\
\hline SBP Z score & $0.385^{* *}$ & $0.389^{* *}$ & $0.433^{* *}$ & $0.198^{*}$ \\
\hline DBP Z score & $0.392^{* *}$ & $0.353^{* *}$ & $0.418^{* *}$ & $0.186^{*}$ \\
\hline $\mathrm{CRP}^{\mathrm{a}}$ & $0.374^{* *}$ & $0.388^{* *}$ & $0.472^{* *}$ & $0.261^{* *}$ \\
\hline Fibrinogen ${ }^{a}$ & $0.341^{* *}$ & $0.316^{* *}$ & $0.375^{* *}$ & $0.193^{*}$ \\
\hline Adiponectin ${ }^{a}$ & $-0.187^{*}$ & $-0.277^{* *}$ & $-0.201^{*}$ & $-0.279^{* *}$ \\
\hline Leptin $^{a}$ & $0.551^{* *}$ & $0.498^{* *}$ & $0.546^{* *}$ & 0.119 \\
\hline Resistin ${ }^{a}$ & $0.229^{*}$ & $0.181^{*}$ & $0.191^{*}$ & 0.064 \\
\hline
\end{tabular}

Data are Pearson's $\mathrm{R}$ (univariate linear analysis) for single biological variables

${ }^{*} P$-value $<0.05 ;{ }^{* *} P$-value $<0.001$

aLog-transformed variables

\section{Prediction of CV risk factors using models adding anthropometric surrogates of body fat distribution to general adiposity measurements}

The initial model including BMI Z Score, age, sex and pubertal status accounted for respectively 7.4, 43.7, 42.7, $41.4,7.9,4.3,18.8,17.5,14.6,19.9,10,50.2$ and $9.5 \%$ of the fasting glucose, insulin, HOMA IR, QUICKI, triglycerides, HDL-cholesterol, SBP Z Score, DBP Z Score, CRP, fibrinogen, adiponectin, leptin and resistin variances.

Adding WHR $\mathrm{Z}$ Score improved fasting insulin $\left(\mathrm{R}^{2}\right.$ : $45.9 \%$; $\left.r_{\text {partial }}^{2} 3.9 \%\right)$, HOMA IR ( $R^{2}: 44.7 \%$; $\left.r_{\text {partial: }}^{2} .6 \%\right)$, QUICKI ( $\mathrm{R}^{2}: 43.3 \%$; $\left.\mathrm{r}_{\text {partial: }}^{2} 3.3 \%\right)$, HDL-cholesterol $\left(\mathrm{R}^{2}\right.$ : $9.6 \%$; $\left.r_{\text {partial }}^{2} 5.6 \%\right)$, triglycerides $\left(R^{2}: 11.7 \%\right.$; $\left.r_{\text {partial }}^{2} 4.2 \%\right)$, adiponectin ( $\mathrm{R}^{2}: 14.3 \%$; $\left.\mathrm{r}_{\text {partial }}^{2} 4.7 \%\right)$ and CRP $\left(\mathrm{R}^{2}: 18.2 \%\right.$; $\left.\mathrm{r}_{\text {partial }}^{2}: 4.3 \%\right)$ prediction.

Associating Waist C Z Score with BMI Z Score, age, sex and pubertal status showed similar findings except for CRP. Indeed, Waist C Z Score accounted for $3.2 \%$ of fasting insulin variance $\left(\mathrm{R}^{2}: 45.5 \%\right)$, respectively for $2.6 \%$ of HOMA IR ( $\left.\mathrm{R}^{2}: 44.2 \%\right), 2.5 \%$ of QUICKI $\left(\mathrm{R}^{2}\right.$ : $42.9 \%), 6.8 \%$ of HDL-cholesterol ( $\left.\mathrm{R}^{2}: 10.8 \%\right), 4.7 \%$ of triglycerides $\left(\mathrm{R}^{2}: 12.2 \%\right)$ and $8.5 \%$ of adiponectin $\left(\mathrm{R}^{2}\right.$ : $17.7 \%)$ variances.

Associated with BMI Z Score, age, sex and pubertal status, WHtR accounted for $2.4 \%$ of the HDL-cholesterol variance $\left(R^{2}: 6.5 \%\right)$, respectively for $4.4 \%$ of the SBP $Z$ Score $\left(R^{2}: 22.4 \%\right), 3 \%$ of the DBP Z Score $\left(R^{2}: 20 \%\right)$, $10.2 \%$ of the CRP $\left(R^{2}: 23.3 \%\right)$ and $2.4 \%$ of the fibrinogen $\left(\mathrm{R}^{2}: 21.8 \%\right.$ ) variances (Table 3 ).

Finally, as regards DXA measurements, apart from fasting glucose, LDL cholesterol, fibrinogen and leptin, the DXA prediction of every other cardiometabolic risk factor was improved when the trunk/legs fat mass index was added to total fat mass, as well as after the addition of trunk fat mass to total fat mass (models were adjusted on age, sex and pubertal status) (Table 4).

\section{Discussion}

Our study clearly showed that, in addition to global overweight and obesity, body fat distribution, as assessed by anthropometry, significantly and independently contributes to the prediction of $\mathrm{CV}$ risk factors in overweight and obese youth. Insulin resistance markers, in particular, were more accurately predicted by adding WHR Z Score or Waist C Z Score to BMI Z Score. HDL cholesterol was unanimously more accurately predicted by adding to BMI $\mathrm{Z}$ Score one of the three selected anthropometric surrogates for body fat distribution. Triglyceride concentration was more accurately predicted after adding either WHR Z Score or Waist C Z Score to BMI Z Score. Inflammation, as assessed by $\mathrm{C}$-reactive protein, had its prediction improved when WHR Z Score and/or WHtR were added to BMI Z Score. WHtR played a similar role in the case of fibrinogen. WHtR played a role also in blood pressure prediction, after combination with BMI Z Score. Adiponectin concentrations seem to be better approached by combining WHR or Waist C Z Scores with BMI Z Score, while resistin and leptin predictions were not affected by the anthropometric measures for body fat distribution. This was also the case of glucose concentrations, the prediction of which was not affected beyond BMI neither by WHR and Waist C Z Scores nor by WHtR. On the other hand, our 
Table 3 Multivariable anthropometric prediction of cardiovascular risk factors in youths

\begin{tabular}{|c|c|c|c|c|c|c|c|c|c|c|}
\hline \multirow[t]{2}{*}{ Dependent variable } & \multirow{2}{*}{$\begin{array}{l}\text { Model } 1: \mathrm{BMI}_{\mathrm{z} \text { score }} \\
\mathrm{R}^{2} \text { model } 1\end{array}$} & \multicolumn{3}{|c|}{ Model 2: BMI z score, WHR z score } & \multicolumn{3}{|c|}{ Model 3: BMI z score, Waist $C_{\text {z score }}$} & \multicolumn{3}{|c|}{ Model 4: BMl z score, WHtR } \\
\hline & & $\overline{R^{2} \text { model } 2}$ & $r^{2}$ partial BMl z score & $\mathrm{r}^{2}$ partial WHR z score & $R^{2}$ model 3 & $r^{2}$ partial BMI z score & $r^{2}$ partial waist $C_{z \text { score }}$ & $\mathrm{R}^{2}$ model 4 & $r^{2}$ partial BMI z score & $\begin{array}{l}r^{2} \text { partial } \\
\text { WHtR }\end{array}$ \\
\hline Fasting glucose & $0.074^{*}$ & $0.074^{*}$ & $0.042^{*}$ & 0.000 & $0.080^{*}$ & $0.027^{*}$ & 0.007 & $0.074^{*}$ & 0.014 & 0.000 \\
\hline Fasting insulin $^{a}$ & $0.437^{* *}$ & $0.459^{* *}$ & $0.266^{* *}$ & $0.039^{*}$ & $0.455^{* *}$ & 0.016 & $0.032^{*}$ & $0.440^{* *}$ & $0.071^{* *}$ & 0.005 \\
\hline HOMA IR $\mathbb{R}^{a}$ & $0.427^{* *}$ & $0.447^{* *}$ & $0.262^{* *}$ & $0.036^{*}$ & $0.442^{* *}$ & 0.019 & $0.026^{*}$ & $0.430^{* *}$ & $0.070^{* *}$ & 0.005 \\
\hline QUICKI & $0.414^{* *}$ & $0.433^{* *}$ & $0.242^{* *}$ & $0.033^{*}$ & $0.429^{* *}$ & 0.016 & $0.025^{*}$ & $0.416^{* *}$ & $0.066^{* *}$ & 0.004 \\
\hline Triglycerides $^{a}$ & $0.079^{*}$ & $0.117^{* *}$ & $0.033^{*}$ & $0.042^{*}$ & $0.122^{* *}$ & 0.009 & $0.047^{*}$ & $0.095^{*}$ & 0.000 & 0.017 \\
\hline HDL cholesterol ${ }^{\mathrm{a}}$ & $0.043^{*}$ & $0.096^{*}$ & $0.022^{*}$ & $0.056^{* *}$ & $0.108^{* *}$ & $0.023^{*}$ & $0.068^{* *}$ & $0.065^{*}$ & 0.001 & $0.024^{*}$ \\
\hline LDL cholesterol & 0.011 & 0.013 & 0.002 & 0.002 & 0.015 & 0.005 & 0.004 & 0.018 & 0.009 & 0.007 \\
\hline SBP Z score & $0.188^{* *}$ & $0.200^{* *}$ & $0.140^{* *}$ & 0.014 & $0.201^{* *}$ & 0.007 & 0.016 & $0.224^{* *}$ & 0.003 & $0.044^{*}$ \\
\hline DBP Z score & $0.175^{* *}$ & $0.184^{* *}$ & $0.130^{* *}$ & 0.010 & $0.176^{* *}$ & $0.028^{*}$ & 0.000 & $0.200^{* *}$ & 0.006 & $0.030^{*}$ \\
\hline $\mathrm{CRP}^{\mathrm{a}}$ & $0.146^{* *}$ & $0.182^{* *}$ & $0.113^{* *}$ & $0.043^{*}$ & $0.158^{* *}$ & 0.005 & 0.014 & $0.233^{* *}$ & 0.004 & $0.102^{* *}$ \\
\hline Fibrinogen ${ }^{a}$ & $0.199^{* *}$ & $0.208^{* *}$ & $0.116^{* *}$ & 0.012 & $0.199^{* *}$ & $0.021^{*}$ & 0.001 & $0.218^{* *}$ & 0.006 & $0.024^{*}$ \\
\hline Adiponectin $^{a}$ & $0.100^{* *}$ & $0.143^{* *}$ & 0.011 & $0.047^{*}$ & $0.177^{* *}$ & $0.040^{*}$ & $0.085^{* *}$ & $0.107^{* *}$ & 0.000 & 0.007 \\
\hline Leptin $^{a}$ & $0.502^{* *}$ & $0.502^{* *}$ & $0.412^{* *}$ & 0.001 & $0.502^{* *}$ & $0.111^{* *}$ & 0.001 & $0.511^{* *}$ & $0.101^{* *}$ & 0.019 \\
\hline Resistin $^{a}$ & $0.095^{* *}$ & $0.095^{*}$ & $0.044^{*}$ & 0.000 & $0.097^{*}$ & 0.017 & 0.002 & $0.095^{*}$ & 0.016 & 0.000 \\
\hline
\end{tabular}

All models were age, sex and pubertal status adjusted

" $P$-value $<0.05$; " $P$-value $<0.001$

a Log-transformed variables 
Table 4 Multivariable DXA prediction of cardiovascular risk factors in youths

\begin{tabular}{|c|c|c|c|c|c|c|c|}
\hline \multirow[t]{2}{*}{ Dependent variable } & \multirow{2}{*}{$\begin{array}{l}\text { Model } 1 \text { : total fat mass } \\
\mathrm{R}^{2} \text { model } 1\end{array}$} & \multicolumn{3}{|c|}{ Model 2: total fat mass, trunk/legs fat mass } & \multicolumn{3}{|c|}{ Model 3: total fat mass, trunk fat mass } \\
\hline & & $\mathrm{R}^{2}$ model 2 & $r^{2}$ partial total fat mass & $r^{2}$ partial trunk/legs fat mass & $\mathrm{R}^{2}$ model 3 & $r^{2}$ partial total fat mass & $r^{2}$ partial trunk fat mass \\
\hline Fasting glucose & $0.058^{*}$ & $0.058^{*}$ & $0.029^{*}$ & 0.000 & $0.058^{*}$ & 0.004 & 0.000 \\
\hline Fasting insulin $^{a}$ & $0.376^{* *}$ & $0.425^{* *}$ & $0.249^{* *}$ & $0.078^{* *}$ & $0.395^{* *}$ & 0.000 & $0.030^{*}$ \\
\hline HOMA $\mathbb{R}^{\mathrm{a}}$ & $0.366^{* *}$ & $0.412^{* *}$ & $0.242^{* *}$ & $0.073^{* *}$ & $0.383^{* *}$ & 0.000 & $0.027^{*}$ \\
\hline QUICKI & $0.349^{* *}$ & $0.394^{* *}$ & $0.213^{* *}$ & $0.069^{* *}$ & $0.365^{* *}$ & 0.000 & $0.025^{*}$ \\
\hline Triglycerides $^{a}$ & 0.046 & $0.095^{*}$ & $0.029^{*}$ & $0.052^{*}$ & $0.075^{*}$ & 0.016 & $0.031^{*}$ \\
\hline $\mathrm{HDL}$ cholesterol $^{a}$ & 0.015 & $0.105^{* *}$ & 0.014 & $0.091^{* *}$ & $0.066^{*}$ & $0.037^{*}$ & $0.051^{*}$ \\
\hline LDL cholesterol & 0.010 & 0.011 & 0.005 & 0.001 & 0.010 & 0.001 & 0.000 \\
\hline SBP Z score & $0.201^{* *}$ & $0.226^{* *}$ & $0.183^{* *}$ & $0.032^{*}$ & $0.230^{* *}$ & 0.004 & $0.037^{*}$ \\
\hline DBP Z score & $0.144^{* *}$ & $0.178^{* *}$ & $0.127^{* *}$ & $0.039^{*}$ & $0.181^{* *}$ & 0.010 & $0.042^{*}$ \\
\hline $\mathrm{CRP}^{\mathrm{a}}$ & $0.165^{* *}$ & $0.198^{* *}$ & $0.164^{* *}$ & $0.039^{*}$ & $0.186^{* *}$ & 0.001 & $0.025^{*}$ \\
\hline Fibrinogen $^{a}$ & $0.216^{* *}$ & $0.216^{* *}$ & $0.158^{* *}$ & 0.000 & $0.217^{* *}$ & 0.018 & 0.000 \\
\hline Adiponectin $^{\mathrm{a}}$ & $0.077^{*}$ & $0.137^{* *}$ & 0.005 & $0.065^{* *}$ & $0.112^{* *}$ & $0.029^{*}$ & $0.038^{*}$ \\
\hline Leptin $^{a}$ & $0.575^{* *}$ & $0.578^{* *}$ & $0.506^{* *}$ & 0.005 & $0.582^{* *}$ & $0.138^{* *}$ & 0.015 \\
\hline Resistin $^{a}$ & $0.100^{* *}$ & $0.120^{* *}$ & $0.063^{* *}$ & $0.022^{*}$ & $0.120^{* *}$ & 0.006 & $0.023^{*}$ \\
\hline
\end{tabular}

All models were age, sex and pubertal status adjusted

${ }^{*} P$-value $<0.05$; ${ }^{* *} P$-value $<0.001$

a Log-transformed variables 
findings based on anthropometric measures were in coherence with the associations observed between the aforementioned CV risk factors and DXA combinations: total fat mass and trunk fat mass; respectively total fat mass and trunk/legs fat mass.

Significant relationships linking unfavourable CV profiles to body fat distribution measures, beyond BMI, have been observed in adults since the pioneer work of Vague. Vague pointed out abdominal fat toxicity to be responsible for severe obesities and serious associated prognosis in adults, in opposition to the gynoid shapes which do not expose to similar hazardous health complications [44]. Since that study, several epidemiological investigations in adults showed in particular that, beyond fatness degrees as assessed by BMI, Waist $\mathrm{C}$ and/or WHR, measuring upper body fat distribution, were significantly correlated with blood pressure, total serum cholesterol, HDL-cholesterol, triglycerides level and/or serum insulin level [30-33].

However, the scarce published studies in children about the usefulness of adding anthropometric surrogates for body fat distribution to BMI remain controversial. Certain American paediatric studies reported, exactly as is shown in the present study, a significant impact of WHR in addition to BMI, to predict HDL-cholesterol and triglycerides, in youth aged 4-19 years [19, 28]. Gillum [18] also showed an improvement in blood pressure prediction in youths $(6-17$ y) by adding WHR to BMI. Maffeis et al. [20] showed significant associations between Waist $C$ and Apo lipoproteins, HDL-cholesterol, total/HDL cholesterol ratio, blood pressure, after BMI, age and sex adjustments in prepubertal children aged 3 to 11 years old.

Nevertheless, in 15-16 year-old youths, Lawlor et al. [15] concluded with the superiority of BMI on Waist C in predicting blood pressure, fasting glucose and insulin, triglycerides, LDL and HDL-cholesterol. Only BMI was also highlighted by Garnett et al. to track CV risk between childhood and adolescence [13]. Likewise, with a view to detecting arterial hypertension in 8-10 year-old children, Maximova et al. recommended the measurement of BMI rather than Waist C or WHtR [45]. Gillum et al. [24] showed no significant differences between BMI and WHR for the prediction of CRP in Mexican American children (6-11 y). Similar abilities of BMI-for-age and WHtR were also shown by Freedman et al. [26] for the screening of fasting insulin, blood pressure, triacylglycerol, HDL, LDL and total-to-HDL cholesterol ratio in the Bogalusa Heart Study.

These controversies may be partly explained by the different methodologies applied in the studies. Actually, some studies used continuous data $[15,18-20,28]$, while others analysed categorical data [13, 24, 26, 27, 45]. Indeed, using categorical rather than continuous data might result in information loss. The lack of standardized international thresholds to define weight status in children (e.g., for normal-weight versus overweight and obesity) may also impact data interpretations. In the current study, we showed different weight status frequencies according to two definitions suggested in the literature: $64 \%$ of obesity and $36 \%$ of overweight according to the IOTF definition [35, 46] and L,M,S Dutch values [42], respectively $80.8 \%$ of obesity and $19.2 \%$ of overweight according to the WHO definition [47]. The lack of a specific national percentile distribution of anthropometric data in youths appears to be an undeniable issue. That constituted a limitation of the current study. However, thanks to the Dutch $\mathrm{L}, \mathrm{M}, \mathrm{S}$ values provided to us by Dr Van Buuren from the Department of Statistics, Quality of Life, Leiden, Netherlands [42, 43], we were able to develop BMI, Waist $\mathrm{C}$ and WHR Z Scores after having checked that Luxembourgish and Dutch paediatric BMI means were similar.

The heterogeneity in the relationships between anthropometry and CV risk factors may also be attributed to the age groups considered in the different studies and/or to the few biological parameters tested. Our study sample was characterized by a broad age range and an exhaustive set of cardiovascular risk factors tested.

The selected nature and relatively small size of our sample, including only overweight and obese subjects, might be a limitation of the current study in that it does not allow the extrapolation of our findings to the general paediatric population. However, as young people who may be at higher risk for $\mathrm{CV}$ impairments are mostly the overweight and obese ones, the current findings might widely apply to this high-risk population subgroup.

\section{Conclusions}

In conclusion, combining BMI Z Score with only one anthropometric measure for regional fat (i.e., WHR Z Score, Waist C Z Score and/or WHtR) improves the prediction of the cardiometabolic, inflammatory and/or adipokines profiles amongst youth. These findings might be useful to inform research and clinical activities, and might help public health authorities to implement a more appropriate and cost-effective screening of overweight, obesity and related comorbidities in youth.

\section{Abbreviations \\ BMl: Body mass index; WHR: Waist-to-hip ratio; Waist C: Waist circumference; WHtR: Waist-to-height ratio; DXA: Dual energy X-ray absorptiometry; HDL-cholesterol: High-density lipoprotein cholesterol; CV: Cardiovascular; SBP: Systolic blood pressure; DBP: Diastolic blood pressure; LDL-cholesterol: Low- density lipoprotein cholesterol; HOMA IR: Homeostasis model assessment of insulin resistance; QUICKI index: Quantitative insulin sensitivity check index; IOTF: International Obesity Task Force.}

Competing interests

The author(s) declare that they have no competing interests.

\section{Authors' contributions}

HS (co) conceived the study and (co) designed the protocol, carried out the anthropometric measurements, interpreted the DXA images, (co) analysed 
the data, interpreted the statistics and drafted the manuscript. CDB (Co) conceived the study and (co) designed the protocol, included the participants and (co)interpreted the statistical analyses. BCG (co)interpreted the statistical analyses and have been involved in drafting the manuscript. GG performed the biological assessment and wrote the biological measurements protocol. MH designed the DXA protocol and managed the DXA collected data. JJ managed and (co) analysed and interpreted the data. MV (co) designed the protocol, calculated the sample size and gave statistical advices. FD (co) conceived the study, (co) designed the protocol and (CO) interpreted the data analyses. SL performed the statistical analyses for the revision of the manuscript. CDB, SS, BCG, SL, MV and FD revised critically the manuscript for important intellectual content. All authors read and approved the final manuscript.

\section{Acknowledgements}

We thank the children and the parents for their participation. We also thank Dr Van Burren (Department of Statistics, TNO Quality of Life, 2301 CE Leiden, The Netherlands) who provided us with the L, M and S values initially developed in the Dutch population.

This study has been funded by the Ministry for Culture, Higher Education and Research, Luxembourg and by the National Research Fund, Luxembourg.

\section{Author details}

${ }^{1}$ Population Health Department, Epidemiology and Public Health Research Unit, Luxembourg Institute of Health, 1A-B, rue Thomas Edison, L-1445 Strassen, Luxembourg. ${ }^{2}$ Centre Hospitalier de Luxembourg, Diabetes \& Endocrinology Care Clinique Pédiatrique (DECCP), L-1210 Luxembourg, Luxembourg. ${ }^{3}$ Faculty for Health engineering and management, UDSL/ILIS, University Lille-Northern France, EA 2694, Laboratory of Public Health, F-59120 Loos, France. ${ }^{4}$ Department of Clinical Biology, Centre Hospitalier de Luxembourg, L-1210 Luxembourg, Luxembourg. ${ }^{5}$ ZithaKlinik, Rheumatology Department, L-2763 Luxembourg, Luxembourg. ${ }^{6}$ Luxembourg Institute of Health, Centre of Competence for Methodology and Statistics (CCMS), L-1445 Strassen, Luxembourg. ${ }^{7}$ Endocrinology and Diabetology Department, Centre Hospitalier de Luxembourg, L-1210 Luxembourg, Luxembourg.

Received: 20 May 2015 Accepted: 13 October 2015

Published online: 24 October 2015

\section{References}

1. Morrison JA, Friedman LA, Wang P, Glueck CJ. Metabolic syndrome in childhood predicts adult metabolic syndrome and type 2 diabetes mellitus 25 to 30 years later. J Pediatr. 2008;152(2):201-6.

2. Grundy SM. Metabolic syndrome: connecting and reconciling cardiovascular and diabetes worlds. J Am Coll Cardiol. 2006;47(6):1093-100.

3. Sabin MA, Magnussen CG, Juonala M, Shield JP, Kahonen M, Lehtimaki T, et al. Insulin and BMI as predictors of adult type 2 diabetes mellitus. Pediatrics. 2015;135(1):e144-51.

4. Cai L, Wu Y, Cheskin L, Wilson RF, Wang Y. Effect of childhood obesity prevention programmes on blood lipids: a systematic review and meta-analysis. Obes Rev. 2014;15(12):933-44.

5. Huang RC, Burrows S, Mori TA, Oddy WH, Beilin L. Lifecourse adiposity and blood pressure between birth and 17 years old. Am J Hypertens. 2015;28(8):1056-63.

6. Athyros VG, Tziomalos K, Karagiannis A, Anagnostis P, Mikhailidis DP. Should adipokines be considered in the choice of the treatment of obesity-related health problems? Curr Drug Targets. 2010;11(1):122-35.

7. Wen X, Pekkala S, Wang R, Wiklund P, Feng G, Cheng SM, et al. Does systemic low-grade inflammation associate with fat accumulation and distribution? A 7-year follow-up study with peripubertal girls. J Clin Endocrinol Metab. 2014;99(4):1411-9.

8. Ortega L, Riestra P, Navarro P, Gavela-Perez T, Soriano-Guillen L, Garces C. Resistin levels are related to fat mass, but not to body mass index in children. Peptides. 2013;49:49-52.

9. Bosch TA, Dengel DR, Kelly AS, Sinaiko AR, Moran A, Steinberger J. Visceral adipose tissue measured by DXA correlates with measurement by $C T$ and is associated with cardiometabolic risk factors in children. Pediatr Obes. 2015;10(3):172-9.

10. Samouda H, De Beaufort C, Stranges S, Hirsch M, Van Nieuwenhuyse JP, Dooms G, Gilson G, Keunen O, Leite S, Vaillant M et al. Cardiometabolic risk: leg fat is protective during childhood. Pediatr Diabetes. 2015. doi:10.1111/pedi.12292.

11. Bauer J, Thornton J, Heymsfield S, Kelly K, Ramirez A, Gidwani S, et al. Dual-energy X-ray absorptiometry prediction of adipose tissue depots in children and adolescents. Pediatr Res. 2012;72(4):420-5.

12. Bigornia SJ, LaValley MP, Benfield LL, Ness AR, Newby PK. Relationships between direct and indirect measures of central and total adiposity in children: what are we measuring? Obesity (Silver Spring, Md). 2013;21(10):2055-62.

13. Garnett SP, Baur LA, Srinivasan S, Lee JW, Cowell CT. Body mass index and waist circumference in midchildhood and adverse cardiovascular disease risk clustering in adolescence. Am J Clin Nutr. 2007;86(3):549-55.

14. Jung C, Fischer N, Fritzenwanger M, Figulla HR. Anthropometric indices as predictors of the metabolic syndrome and its components in adolescents. Pediatr Int. 2010;52(3):402-9.

15. Lawlor DA, Benfield L, Logue J, Tilling K, Howe LD, Fraser A, et al. Association between general and central adiposity in childhood, and change in these, with cardiovascular risk factors in adolescence: prospective cohort study. BMJ (Clinical research ed). 2010;341:c6224.

16. Weber DR, Levitt Katz LE, Zemel BS, Gallagher PR, Murphy KM, Dumser SM, et al. Anthropometric measures of abdominal adiposity for the identification of cardiometabolic risk factors in adolescents. Diabetes Res Clin Pract. 2014;103(3):e14-7.

17. Grober-Gratz D, Widhalm K, de Zwaan M, Reinehr T, Bluher S, Schwab KO, et al. Body mass index or waist circumference: which is the better predictor for hypertension and dyslipidemia in overweight/obese children and adolescents? Association of cardiovascular risk related to body mass index or waist circumference. Horm Res Paediatr. 2013;80(3):170-8.

18. Gillum RF. The association of the ratio of waist to hip girth with blood pressure, serum cholesterol and serum uric acid in children and youths aged 6-17 years. J Chronic Dis. 1987:40(5):413-20.

19. Gillum RF. Distribution of waist-to-hip ratio, other indices of body fat distribution and obesity and associations with HDL cholesterol in children and young adults aged 4-19 years: the Third National Health and Nutrition Examination Survey. Int J Obes Relat Metab Disord. 1999;23(6):556-63.

20. Maffeis C, Pietrobelli A, Grezzani A, Provera S, Tato L. Waist circumference and cardiovascular risk factors in prepubertal children. Obes Res. 2001;9(3):179-87.

21. Griffiths C, Gately P, Marchant PR, Cooke CB. Cross-sectional comparisons of BMI and waist circumference in British children: mixed public health messages. Obesity (Silver Spring, Md). 2012;20(6):1258-60.

22. Browning LM, Hsieh SD, Ashwell M. A systematic review of waist-to-height ratio as a screening tool for the prediction of cardiovascular disease and diabetes: 0.5 could be a suitable global boundary value. Nutr Res Rev. 2010;23(2):247-69.

23. Taylor RW, Williams SM, Grant AM, Taylor BJ, Goulding A. Predictive ability of waist-to-height in relation to adiposity in children is not improved with age and sex-specific values. Obesity (Silver Spring, Md). 2011;19(5):1062-8.

24. Gillum RF. Association of serum C-reactive protein and indices of body fat distribution and overweight in Mexican American children. J Natl Med Assoc. 2003;95(7):545-52.

25. Katzmarzyk PT, Srinivasan SR, Chen W, Malina RM, Bouchard C, Berenson GS. Body mass index, waist circumference, and clustering of cardiovascular disease risk factors in a biracial sample of children and adolescents. Pediatrics. 2004;114(2):e198-205.

26. Freedman DS, Kahn HS, Mei Z, Grummer-Strawn LM, Dietz WH, Srinivasan $\mathrm{SR}$, et al. Relation of body mass index and waist-to-height ratio to cardiovascular disease risk factors in children and adolescents: the Bogalusa Heart Study. Am J Clin Nutr. 2007;86(1):33-40.

27. Savva SC, Tornaritis M, Savva ME, Kourides Y, Panagi A, Silikiotou N, et al. Waist circumference and waist-to-height ratio are better predictors of cardiovascular disease risk factors in children than body mass index. Int J Obes Relat Metab Disord. 2000;24(11):1453-8.

28. Gillum RF. Indices of adipose tissue distribution, apolipoproteins B and Al, lipoprotein (a), and triglyceride concentration in children aged 4-11 years: the Third National Health and Nutrition Examination Survey. J Clin Epidemiol. 2001;54(4):367-75.

29. Huang RC, de Klerk N, Mori TA, Newnham JP, Stanley FJ, Landau LI, et al. Differential relationships between anthropometry measures and cardiovascular risk factors in boys and girls. Int J Pediatr Obes. 2011;6(2-2):e271-82.

30. Larsson B, Svardsudd K, Welin L, Wilhelmsen L, Bjorntorp P, Tibblin G. Abdominal adipose tissue distribution, obesity, and risk of cardiovascular 
disease and death: 13 year follow up of participants in the study of men born in 1913. Br Med J (Clin Res Ed). 1984;288(6428):1401-4.

31. Rexrode KM, Carey VJ, Hennekens CH, Walters EE, Colditz GA, Stampfer MJ, et al. Abdominal adiposity and coronary heart disease in women. JAMA. 1998;280(21):1843-8

32. Seidell JC, Cigolini M, Charzewska J, Ellsinger BM, di Biase G. Fat distribution in European women: a comparison of anthropometric measurements in relation to cardiovascular risk factors. Int J Epidemiol. 1990;19(2):303-8.

33. Seidell JC, Cigolini M, Deslypere JP, Charzewska J, Ellsinger BM, Cruz A. Body fat distribution in relation to serum lipids and blood pressure in 38-year-old European men: the European fat distribution study. Atherosclerosis. 1991;86(2-3):251-60.

34. Pischon T, Boeing H, Hoffmann K, Bergmann M, Schulze MB, Overvad K, et al. General and abdominal adiposity and risk of death in Europe. N Engl J Med. 2008;359(20):2105-20.

35. Cole TJ, Bellizzi MC, Flegal KM, Dietz WH. Establishing a standard definition for child overweight and obesity worldwide: international survey. BMJ (Clin Res Ed). 2000;320(7244):1240-3.

36. Lohmann T, Roche A, Martorell R. In: Edited by (Ed) Cl: Human Kinetics Books. Anthropometric standardization reference manual. 1988.

37. National High Blood Pressure Education Program Working Group on High Blood Pressure in C, Adolescents. The fourth report on the diagnosis, evaluation, and treatment of high blood pressure in children and adolescents. Pediatrics. 2004;114(2 Suppl 4th Report):555-76.

38. Turner RC, Holman RR, Matthews D, Hockaday TD, Peto J. Insulin deficiency and insulin resistance interaction in diabetes: estimation of their relative contribution by feedback analysis from basal plasma insulin and glucose concentrations. Metabolism. 1979;28(11):1086-96.

39. Chen H, Sullivan G, Quon MJ. Assessing the predictive accuracy of QUICKI as a surrogate index for insulin sensitivity using a calibration model. Diabetes. 2005;54(7):1914-25.

40. Marshall WA, Tanner JM. Variations in pattern of pubertal changes in girls. Arch Dis Child. 1969:44(235):291-303.

41. Marshall WA, Tanner JM. Variations in the pattern of pubertal changes in boys. Arch Dis Child. 1970;45(239):13-23.

42. Fredriks AM, van Buuren S, Wit JM, Verloove-Vanhorick SP. Body index measurements in 1996-7 compared with 1980. Arch Dis Child. 2000;82(2):107-12.

43. Fredriks AM, van Buuren S, Fekkes M, Verloove-Vanhorick SP, Wit JM. Are age references for waist circumference, hip circumference and waist-hip ratio in Dutch children useful in clinical practice? Eur J Pediatr. 2005;164(4):216-22.

44. Vague J. The degree of masculine differentiation of obesities: a factor determining predisposition to diabetes, atherosclerosis, gout, and uric calculous disease. Am J Clin Nutr. 1956;4(1):20-34.

45. Maximova K, Chiolero A, O'Loughliin J, Tremblay A, Lambert M, Paradis G. Ability of different adiposity indicators to identify children with elevated blood pressure. J Hypertens. 2011;29(11):2075-83.

46. Cole TJ. Software for LMS method. http:// Imschartmaker.software.informer.com/2.5/

47. de Onis M, Onyango AW, Borghi E, Siyam A, Nishida C, Siekmann J. Development of a WHO growth reference for school-aged children and adolescents. Bull World Health Organ. 2007;85(9):660-7.

\section{Submit your next manuscript to BioMed Central and take full advantage of:}

- Convenient online submission

- Thorough peer review

- No space constraints or color figure charges

- Immediate publication on acceptance

- Inclusion in PubMed, CAS, Scopus and Google Scholar

- Research which is freely available for redistribution 